\title{
On families of differential equations on two-torus with all phase-lock areas
}

\author{
Alexey Glutsyuk ${ }^{* \dagger \ddagger}$ Leonid Rybnikov ${ }^{\S}$
}

June 11, 2018

\begin{abstract}
We consider two-parametric families of non-autonomous ordinary differential equations on the two-torus with the coordinates $(x, t)$ of the type $\dot{x}=v(x)+A+B f(t)$. We study its rotation number as a function of the parameters $(A, B)$. The phase-lock areas are those level sets of the rotation number function $\rho=\rho(A, B)$ that have nonempty interiors. V.M.Buchstaber, O.V.Karpov, S.I.Tertychnyi have studied the case, when $v(x)=\sin x$ in their joint paper. They have observed the quantization effect: for every smooth periodic function $f(t)$ the family of equations may have phase-lock areas only for integer rotation numbers. Another proof of this quantization statement was later obtained in a joint paper by Yu.S.Ilyashenko, D.A.Filimonov, D.A.Ryzhov. This implies the similar quantization effect for every $v(x)=a \sin (m x)+b \cos (m x)+c$ and rotation numbers that are multiples of $\frac{1}{m}$. We show that for every other analytic vector field $v(x)$ (i.e., having at least two Fourier harmonics with non-zero non-opposite degrees and nonzero coefficients) there exists an analytic periodic function $f(t)$ such that the corresponding family of equations has phaselock areas for all the rational values of the rotation number.
\end{abstract}

\footnotetext{
${ }^{*}$ CNRS, France (UMR 5669 (UMPA, ENS de Lyon) and UMI 2615 (Lab. J.V.Poncelet)). E-mail: aglutsyu@ens-lyon.fr

${ }^{\dagger}$ National Research University Higher School of Economics (HSE), Moscow, Russia

${ }^{\ddagger}$ Supported by part by RFBR grants 13-01-00969-a, 16-01-00748, 16-01-00766 and by ANR grant ANR-13-JS01-0010.

${ }^{\S}$ National Research University Higher School of Economics (HSE), International Laboratory of Representation Theory and Mathematical Physics, 20 Myasnitskaya st, Moscow 101000, Russia, and Institute for Information Transmission Problems, Moscow, Russia. Email: leo.rybnikov@gmail.com

I The work of L.R. was done at IITP under support of a grant from the Russian Science Foundation (project? 14-50-00150).
} 


\section{Introduction}

\subsection{Main result}

We consider families of ordinary differential equations on the torus $\mathbb{T}^{2}=\mathbb{R}^{2}$ / $2 \pi \mathbb{Z}^{2}$ with the coordinates $(x, t)$ of the type

$$
\dot{x}=v(x)+A+B f(t) ; A, B \in \mathbb{R} \text { are the parameters. }
$$

Family (1.1) with $v(x)=\nu \sin x, f(t)=\cos t$ arises in several models in physics, mechanics and geometry. For example, it describes the overdamped model of the Josephson junction (RSJ - model) in superconductivity (our main motivation), see [12, 17, 14, 3, 16]; it arises in planimeters, see [7, 8].

The flow map for the period $2 \pi$ of equation (1.1) is a circle diffeomorphism depending on the parameters $(A, B)$. We study its rotation number $\rho=\rho(A, B)$ as a function of the parameters $A$ and $B$. (Normalization convention: the rotation number of a rotation equals the angle of rotation divided by $2 \pi$.) The $r$-th phase-lock area is the level set

$$
\{(A, B) \mid \rho(A, B)=r\} \subset \mathbb{R}^{2}
$$

provided it has a non-empty interior.

In 2001 J.Guckenheimer and Yu.S.Ilyashenko [9] studied the family

$$
\dot{x}=\varepsilon^{-1}(a-\cos x-\cos t)
$$

as a slow-fast system with small parameter $\varepsilon>0$. They observed the following new effect: there exists a sequence of intervals $C_{n} \subset\{\varepsilon>0\}, C_{n} \rightarrow 0$ as $n \rightarrow \infty$, such that for every $\varepsilon \in C_{n}$ equation (1.2) has an attracting canard limit cycle. They have conjectured that this effect observed in a very special family (1.2) should be observed in a generic slow-fast system on two-torus.

In the same year 2001 the family of equations (1.1) with $v(x)=\sin x$, $f(t)=\sin t$ similar to (1.2) was studied by V.M.Buchstaber, O.V.Karpov, S.I.Tertychnyi 44 from a different point of view. They have observed the following quantization effect of the rotation number. Another proof of this quantization statement was later obtained in [11, 10].

Theorem 1.1 [4, 11, 10] Let

$$
v(x)=a \sin m x+b \cos m x+c, a, b, c \in \mathbb{R}, m \in \mathbb{Z} .
$$

Then for every smooth function $f: S^{1} \rightarrow \mathbb{R}$ the corresponding family of equations (1.1) may have phase-lock areas only for those values of the rotation number that are integer multiples of $\frac{1}{m}$. 
Remark 1.2 This theorem was stated and proved in loc.cit. for $m=1$. Its generalization to arbitrary integer values of $m$ then follows immediately by passing to the quotient of the circle by the group of rotations by angles $\frac{2 \pi k}{m}$, $k \in \mathbb{Z}$. The quotient projection transforms a field $v(x)$ as in (1.3) into the corresponding field with $m=1$.

Our main result is the theorem below showing that for any other analytic vector field $v$ the statement of Theorem 1.1 becomes drastically false.

Theorem 1.3 Let $v$ be an analytic vector field on the circle $S^{1}=\mathbb{R} / 2 \pi \mathbb{Z}$ different from (1.3). Then there exists an analytic function $f: S^{1} \rightarrow \mathbb{R}$ such that the corresponding family of differential equations (1.1) has phase-lock areas for all the rational values of the rotation number.

Remark 1.4 A vector field on $S^{1}$ is not of type (1.3), if and only if its Fourier series has at least two harmonics of nonzero non-opposite degrees with nonzero coefficients:

$$
v(x)=\sum_{k} a_{k} e^{i k x}, a_{k} \in \mathbb{C} ; a_{l}, a_{n} \neq 0 \text { for some } l, n \neq 0, l \neq \pm n .
$$

Remark 1.5 Each phase-lock area intersects each horizontal line $B=$ const by a segment (or a point). This follows from continuity of the rotation number and its monotonicity in $A$ (see Proposition 1.10 below). If the field $v$ is analytic, then for every given small $\varepsilon>0$ the intersections of the phaselock areas with the strip $|B|<\varepsilon$ also have non-empty interiors and are usually called the Arnold tongues. The classical Arnold tongue picture for the Arnold family of circle diffeomorphisms can be found in [1, p. 110]. The statement of Theorem 1.3 implies that the corresponding family of equations restricted to the latter strip has Arnold tongues for all the rational values of the rotation number.

\subsection{Plan of the paper and sketch of proof of Theorem 1.3}

The definition and basic properties of the rotation number are recalled in the next subsection. Theorem 1.3 is proved in Section 2 .

Everywhere below for a vector field $w$ by $g_{w}^{t}$ we denote its time $t$ flow map. By $\left(g_{w_{1}}^{t}\right)_{*} w_{2}$ we denote the image of the field $w_{2}$ under the time $t$ flow map of the field $w_{1}$.

Let $v$ be a vector field on $S^{1}$. Denote $m=m(v)$ the greatest common divisor of the degrees of its Fourier harmonics with non-zero coefficients:

$$
m(v)=\text { G.C.D. }\left\{k \in \mathbb{Z} \backslash 0 ; a_{k} \neq 0\right\} \in \mathbb{N} \text {, see (1.4). }
$$


We say that $v$ has coprime harmonics, if $m(v)=1$.

Remark 1.6 The group $\mathbb{Z}_{m}$ acts on $S^{1}$ by rotations of order $m$. For $m=$ $m(v)$ the quotient projection $S^{1} \mapsto S_{m}^{1}=S^{1} / \mathbb{Z}_{m}$ transforms the field $v$ to that with coprime harmonics: it divides the degrees of its harmonics by $m$.

Let $v$ be a vector field that is not of type (1.3). Without loss of generality we consider that $v$ has coprime harmonics: one can achieve this by passing to the above quotient. Let $\Lambda$ denote the Lie algebra generated by the constant vector field $\frac{d}{d x}$, the commutator $v^{\prime}=\left[\frac{d}{d x}, v\right]$ and its images under all the circle rotations.

Theorem 1.3 and its proof are motivated by the following theorem proved in Subsection 2.2.

Theorem 1.7 For every $C^{\infty}$-smooth vector field $v$ on $S^{1}$ having coprime harmonics there is the following alternative:

- either $v(x)=a \sin x+b \cos x+c$ and the Lie algebra $\Lambda$ coincides with that of Möbius group $P S L_{2}(\mathbb{R})$ of conformal automorphisms of the unit disk;

- or $\Lambda$ is $C^{\infty}$ - dense in the space $\chi\left(S^{1}\right)$ of $C^{\infty}$ vector fields on $S^{1}$.

Thus, in our case, when $v$ is not of type (1.3),$\Lambda$ is dense in $\chi\left(S^{1}\right)$.

Remark 1.8 A theorem of Sophus Lie [13] says that every Lie algebra of smooth germs at 0 of vector fields on $\mathbb{R}$ is either at most three-dimensional, or infinite-dimensional (see a recent proof in [15, p. 132]). Some classes of infinite-dimensional Lie algebras of germs of vector fields in any dimension were classified by Elie Cartan, see [6] for references and related results on filtered Lie algebras. A related result on actions of groups of circle diffeomorphisms on tuples of points in terms of the group structure was obtained in [15, theorem A, p. 125]. Specialists believe that Theorem 1.7 is known, but we have not found it in literature.

Let $\operatorname{Dif} f_{+}^{\infty}\left(S^{1}\right)$ denote the group of positive (i.e., orientation-preserving) circle diffeomorphisms. Let $G=G(v) \subset D i f f_{+}^{\infty}\left(S^{1}\right)$ denote the group generated by flows of the vector fields $\frac{d}{d x}$ and $v$. It consists of products

$$
g\left(t_{1}, \ldots, t_{k}, \tau_{1}, \ldots, \tau_{k}\right)=g_{\frac{d}{d x}}^{t_{1}} \circ g_{v}^{\tau_{1}} \circ \cdots \circ g_{\frac{d}{d x}}^{t_{k}} \circ g_{v}^{\tau_{k}} .
$$

Set

$$
\begin{gathered}
G^{s}=\left\{g\left(t_{1}, \ldots, \tau_{k}\right) \in G \mid \sum_{j} \tau_{j}=2 \pi s\right\} \\
G_{+}^{1}=\left\{g\left(t_{1}, \ldots, \tau_{k}\right) \in G^{1} \mid \tau_{j}>0 \text { for all } j\right\} .
\end{gathered}
$$


Definition 1.9 A circle diffeomorphism $\phi: S^{1} \rightarrow S^{1}$ belongs to the class $F(v)$, if it can be realized as a period $2 \pi$ flow map of a differential equation of the type (1.1) with $f(t)$ being a trigonometric polynomial.

Recall that a $q$-periodic point $x_{0}$ of a positive circle diffeomorphism $f$ is hyperbolic, if its multiplier $\left(f^{q}\right)^{\prime}\left(x_{0}\right)$ is different from 1 ; then its periodic orbit is also called hyperbolic. A positive circle diffeomorphism is hyperbolic, if it has periodic orbits and all of them are hyperbolic.

For any family of differential equations depending on parameters analytically, the condition that the corresponding flow map has a given rational rotation number and at least one hyperbolic periodic orbit is an open condition on the parameters. Thus, to prove Theorem 1.3, we have to show that for every $\rho \in \mathbb{Q}$ there exists at least some class $\mathrm{F}(\mathrm{v})$ diffeomorphism with the rotation number $\rho$ and at least one hyperbolic orbit.

The proof of Theorem 1.3 is done as follows.

Step 1. We show (Proposition 2.1 in Subsection 2.1) that the class $F(v)$ diffeomorphisms accumulate to all of $G_{+}^{1}$.

Step 2. We show that the set $G^{1} \supset G_{+}^{1}$ is $C^{\infty}$-dense in $\operatorname{Diff} f_{+}^{\infty}\left(S^{1}\right)$ (Corollary 2.77). This basically follows from density of the Lie algebra $\Lambda$ (Theorem 1.7). In more detail, the density of the algebra $\Lambda$ implies that the group $\mathcal{F}$ generated by the flows of its generators $\frac{d}{d x}$ and $\left[\frac{d}{d x}, v\right]$ is dense (Theorem 2.5). This implies that the group $G^{0}$ is dense, since its closure contains all of $\mathcal{F}$ (Proposition 2.6). This immediately implies density of $G^{1}$.

Step 3. We show (Lemma 2.8 in Subsection 2.3) that for every rational number $\frac{p}{q}$ the set $G_{+}^{1}$ contains a diffeomorphism with rotation number $\frac{p}{q}$ and at least one hyperbolic periodic orbit (either attractor, or repeller). We already know (step 2) that $G^{1}$ is dense and hence, contains hyperbolic circle diffeomorphisms with all the rotation numbers (since the existence of a hyperbolic orbit with a given rotation number is an open condition). We show that each one of them can be analytically deformed in $G^{1}$ to an element of the set $G_{+}^{1}$ without changing rotation number and keeping one and the same point periodic. The multiplier of the corresponding periodic orbit will be an analytic function of the deformation parameters that is not identically equal to one. Thus, we can achieve that the deformed element in $G_{+}^{1}$ have the given rotation number and a hyperbolic orbit. This will prove Lemma 2.8. This is the main place where we use the analyticity of the field $v$.

Step 4. Constructing families (1.1) with all phase-lock areas. Proposition 2.1 and Lemma 2.8 together imply that for every $\frac{p}{q} \in \mathbb{Q}$ there exists a trigonometric polynomial $f(t)$ for which the corresponding family of equa- 
tions (1.1) has phase-lock area with rotation number $\frac{p}{q}$. We then deduce that for every $\frac{p}{q}$ there exists an $N=N\left(\frac{p}{q}\right)$ such that for a generic 1 trigonometric polynomial $f(t)$ of degree at most $N$ the corresponding family of equations (1.1) has phase-lock area corresponding to the rotation number value $\frac{p}{q}$. This together with persistence of phase-lock areas under small perturbations shows that for an appropriate analytic function $f: S^{1} \rightarrow \mathbb{R}$ the corresponding family (1.1) has phase-lock areas for all the rational values of the rotation number. This will prove Theorem 1.3 .

\subsection{Rotation numbers and phase-lock areas}

Let $\mathbb{T}^{2}=\mathbb{R}^{2} / 2 \pi \mathbb{Z}^{2}$ be a torus with coordinates $(x, t)$. Consider the flow given by the nonautonomous differential equation

$$
\dot{x}=\frac{d x}{d t}=\phi(x, t)
$$

with smooth right-hand side. The time $t$ flow mapping is a diffeomorphism $h_{t}: S^{1} \rightarrow S^{1}$ of the space circle. Consider the universal covering

$$
\mathbb{R} \rightarrow S^{1}=\mathbb{R} / 2 \pi \mathbb{Z}
$$

over the space circle. The flow mappings of equation (1.7) can be lifted to the universal covering and induce a smooth family of diffeomorphisms

$$
H_{r, t}: \mathbb{R} \times\{r\} \rightarrow \mathbb{R} \times\{r+t\}, H_{r, 0}=I d .
$$

Recall that for every $(x, r) \in \mathbb{R} \times S^{1}$ there exists a limit

$$
\rho=\lim _{n \rightarrow+\infty} \frac{1}{2 \pi n} H_{r, 2 \pi n}(x) \in \mathbb{R},
$$

which depends neither on $r$, nor on $x$ and is called the rotation number of the flow of equation (1.7) (e.g., see [1, p. 104]).

Now consider an arbitrary analytic family of equations

$$
\dot{x}=\psi(x, t, B)+A,(x, t) \in \mathbb{T}^{2}, A, B \in \mathbb{R} .
$$

Proposition 1.10 [1, pp. 104, 109] The rotation number $\rho=\rho(A, B)$ of the flow of equation (1.9) is a continuous function of the parameters $(A, B)$

\footnotetext{
${ }^{1}$ Everywhere in the paper by "generic" we mean "topologically generic": belonging to an open dense subset.
} 
and a nondecreasing function of $A$. If, for some parameter value, the flow mapping $h_{2 \pi}=h_{A, B, 2 \pi}: S^{1} \times\{0\} \rightarrow S^{1} \times\{0\}$ of equation (1.9) has a periodic orbit of period $q$, and the cyclic order of the orbit on the circle is the same as for an orbit of the rotation $x \mapsto x+\frac{p}{q}, p \in \mathbb{Z}$, then the rotation number is equal to $\frac{p}{q}(\bmod \mathbb{Z})$.

Remark 1.11 The rotation number of an orientation preserving diffeomorphism $h: S^{1} \rightarrow S^{1}, S^{1}=\mathbb{R} / 2 \pi$ is defined analogously. Let us consider its lifting $H: \mathbb{R} \rightarrow \mathbb{R}$ to the universal cover, which is uniquely defined up to post-composition with translation by an integer multiple of $2 \pi$. The limit $\rho=\lim _{n \rightarrow+\infty} \frac{1}{2 \pi n} H^{n}(x)$ exists. It is independent on $x$, well-defined modulo $\mathbb{Z}$ and is called the rotation number $\rho=\rho(h)$, see the same chapter in loc.cit. The rotation number of the time $2 \pi$ flow mapping of a differential equation (1.7) coincides modulo $\mathbb{Z}$ with the rotation number of the equation.

\section{Realization of phase-lock areas: Proof of Theo- rem 1.3}

\subsection{Approximations of products of flows of vector fields by flows of periodic equations}

Proposition 2.1 For every smooth vector field $v$ on $S^{1}$ the corresponding subset $G_{+}^{1} \subset D$ if $f_{+}^{\infty}\left(S^{1}\right)$ from (1.6) is contained in the $C^{\infty}$-closure of class $F(v)$ diffeomorphisms. In more detail, consider an arbitrary $g \in G_{+}^{1}$, let $\rho=\rho(g)$ denote its rotation number. Then for every $\alpha \equiv \rho(\bmod \mathbb{Z}), k \in \mathbb{N}$, $\varepsilon>0$ there exist a trigonometric polynomial $f(t)$ such that the corresponding equation (1.1) with $A=0, B=1$ has rotation number $\alpha$ and its time $2 \pi$ flow map is $\varepsilon$-close to $g$ in the $C^{k}$ topology.

Proof Fix a diffeomorphism

$$
g=g\left(t_{1}, \ldots, t_{k}, \tau_{1}, \ldots, \tau_{k}\right)=g_{\frac{d}{d x}}^{t_{1}} \circ g_{v}^{\tau_{1}} \circ \cdots \circ g_{\frac{d}{d x}}^{t_{k}} \circ g_{v}^{\tau_{k}} ; \tau_{j}>0 ; \sum_{j} \tau_{j}=2 \pi .
$$

Let $\rho \in S^{1}=\mathbb{R} / \mathbb{Z}$ denote its rotation number. Fix an arbitrary $\alpha \equiv$ $\rho(\bmod \mathbb{Z})$. Let us construct a trigonometric polynomial $f(t)$ such that the flow of the corresponding equation (1.1) with $A=0, B=1$ approximates $g$ and has rotation number $\alpha$. Without loss of generality we assume that $t_{j} \neq 0$. For every $s \in \mathbb{Z}, 0 \leq s \leq k$, set

$$
T_{s}=2 \pi-\sum_{j \leq s} \tau_{j} ; 2 \pi=T_{0}>T_{1}>\cdots>T_{k}=0 .
$$


Fix a small $\delta>0$. Let us split the segment $[0,2 \pi]$ into $4 k$ segments

$$
\begin{gathered}
I_{s}=\left[T_{s}, T_{s-1}-\delta-\delta^{2}\right], J_{s}=\left[T_{s-1}-\delta-\delta^{2}, T_{s-1}-\delta\right] ; \\
R_{s}=\left[T_{s-1}-\delta, T_{s-1}-\delta^{2}\right], V_{s}=\left[T_{s-1}-\delta^{2}, T_{s-1}\right] ; s=1, \ldots, k .
\end{gathered}
$$

Set $I_{k+1}=I_{1}, J_{k+1}=J_{1}, R_{k+1}=R_{1}, V_{k+1}=V_{1}$. Note that the segments $I_{j}$ and $R_{s}$ are all disjoint, have size of order at least $\delta$ (for small $\delta$ ) and are separated by segments $J_{p}$ and $V_{i}$ of size $\delta^{2}$. Let us choose a $C^{\infty} 2 \pi$ periodic function $\phi_{\delta}: \mathbb{R} \rightarrow \mathbb{R}$ satisfying the following conditions for every $s=1, \ldots, k$ :

$$
\left.\phi_{\delta}\right|_{I_{s}} \equiv 0,\left.\phi_{\delta}\right|_{R_{s}} \equiv \frac{t_{s}}{\delta}, \phi_{\delta}\left(J_{s} \cup V_{s}\right) \in\left[0, \frac{t_{s}}{\delta}\right] .
$$

More precisely, we choose the function $\phi_{\delta}$ on the segments $J_{s}$ and $V_{s}$ as follows. Fix a $C^{\infty}$ function $\psi:[0,1] \rightarrow[0,1]$ such that $\left.\psi\right|_{\left[0, \frac{1}{3}\right]} \equiv 0,\left.\psi\right|_{\left[\frac{2}{3}, 1\right]} \equiv 1$. Set

$$
\begin{gathered}
\phi_{\delta}(t)=\frac{t_{s}}{\delta} \psi\left(\delta^{-2}\left(t-T_{s-1}+\delta+\delta^{2}\right)\right) \text { for } t \in J_{s} \\
\phi_{\delta}(t)=\frac{t_{s}}{\delta} \psi\left(\delta^{-2}\left(T_{s-1}-t\right)\right) \text { for } t \in V_{s} .
\end{gathered}
$$

Lemma 2.2 The $2 \pi$ time flow mapping of the differential equation

$$
\dot{x}=v(x)+\phi_{\delta}(t)
$$

tends to the mapping $g$ in $C^{\infty}$, as $\delta \rightarrow 0$.

Proof The flow map of equation (2.2) through each segment $I_{s}$ coincides with $g_{v}^{\tau_{s}-\delta-\delta^{2}}$, which tends to $g_{v}^{\tau_{s}}$, as $\delta \rightarrow 0$. The flow through a segment $R_{s}$ tends to $g_{\frac{d}{d x}}^{t_{s}}$. Indeed, let us write down the differential equation in the rescaled time variable $\hat{t}=\delta^{-1}\left(t-T_{s-1}+\delta\right)$. The rescaling transforms the segment $R_{s}$ to $[0,1-\delta]$. The rescaled equation takes the form

$$
\dot{x}=\delta v(x)+t_{s} .
$$

Its flow through the latter segment obviously converges to $g_{\frac{d}{d x}}^{t_{s}}$. Let us show that the flow map of equation (2.2) through each segment $J_{s}$ or $V_{s}$ tends to the identity. Let us prove this statement for a segment $V_{s}$ : the proof for the segments $J_{s}$ is the same. The time rescaling $\hat{t}=\delta^{-2}\left(t-T_{s-1}+\delta^{2}\right)$ transforms $V_{s}$ to the segment $[0,1]$ and equation (2.2) to

$$
\dot{x}=\delta^{2} v(x)+\delta t_{s} \psi(1-\hat{t}) .
$$


Its time one flow mapping obviously converges to the identity together with derivatives, as $\delta \rightarrow 0$. This proves Lemma 2.2 .

The rotation number of equation (2.2) tends to $\beta \equiv \rho(g)(\bmod \mathbb{Z})$, as $\delta \rightarrow 0$, see Lemma 2.2 and its proof. This implies that replacing $t_{1}$ in formula (2.1) by $t_{1}+2 \pi n+\sigma$ with $n=\alpha-\beta \in \mathbb{Z}$ and an appropriate small $\sigma=\sigma(\delta)$, one can achieve that the rotation number of equation (2.2) be equal to the given $\alpha$. The corresponding flow map with thus changed $\phi_{\delta}$ will again converge to $g$. Moreover, we can approximate each $\phi_{\delta}$ by a trigonometric polynomial $f_{\delta}(t)$ so that the flow map of the corresponding equation be close to that of equation (2.2) and the rotation number remains the same. Finally we get a family of analytic differential equations of type (1.1) with $f(t)$ being a trigonometric polynomial such that for $A=0, B=1$ its rotation number equals $\alpha$ and its time $2 \pi$ flow mapping is arbitrarily $C^{\infty}$-close to $g$. Proposition 2.1 is proved.

\subsection{Lie algebra generated by $v$ and $\frac{d}{d x}$ and group generated by their flows}

Let $\mathcal{R} \simeq S^{1}$ denote the group of circle rotations.

Theorem 2.3 Let a $C^{\infty}$ vector field $v$ on $S^{1}$ be not of type (1.3) and have coprime harmonics: $m(v)=1$, see (1.5). Then the Lie algebra $\Lambda$ generated by the vector field $\frac{d}{d x}$, the bracket $v^{\prime}=\left[\frac{d}{d x}, v\right]$ and all the $\mathcal{R}$-images of the field $v^{\prime}$ is dense in the space $\chi\left(S^{1}\right)$ of $C^{\infty}$ vector fields on $S^{1}$.

Remark 2.4 The condition that $\Lambda$ contains the $\mathcal{R}$-images of the bracket $v^{\prime}$ is needed only in the case, when $v$ is non-analytic: if $v$ is analytic, then the latter images are automatically contained in the $C^{\infty}$-closure of the algebra $\Lambda$.

Proof The algebra $\Lambda$ is $\mathcal{R}$-invariant, by definition. The idea of the proof is to study the action of the group $\mathcal{R}$ on a closure of the algebra $\Lambda$ (i.e., its adjoint action) and to split it as a sum of irreducible representations. For every $s \in \mathbb{N}$ let $\bar{\Lambda}_{s}$ denote the closure of the algebra $\Lambda$ in the Sobolev space $H_{s}\left(S^{1}\right)$ of vector fields. The group $\mathcal{R}$ acts on each Sobolev space by unitary transformations, hence the Hilbert space $H_{s}\left(S^{1}\right)$ is naturally a unitary representation of the group $\mathcal{R}$. This representation is completely reducible, i.e. it splits into an orthogonal sum of irreducible representations, as does every unitary representation of the circle. The decomposition of 
$H_{s}\left(S^{1}\right)$ into the direct sum of irreducibles is $H_{s}\left(S^{1}\right)=\bigoplus_{j \in \mathbb{Z}_{\geq 0}} H_{s}\left(S^{1}\right)^{j}$, where $H_{s}\left(S^{1}\right)^{0}=\mathbb{R} \frac{d}{d x}$ is one-dimensional, and $H_{s}\left(S^{1}\right)^{j}=\mathbb{R} \cos j x \frac{d}{d x}+\mathbb{R} \sin j x \frac{d}{d x}$ for $j>0$ are two-dimensional. Note that the irreducible representations $H_{s}\left(S^{1}\right)^{j}$ are pairwise non-isomorphic, and hence each $\mathcal{R}$-invariant subspace in $H_{s}\left(S^{1}\right)$ is the direct sum of some of the $H_{s}\left(S^{1}\right)^{j}$ 's.

Since the Hilbert subspace $\bar{\Lambda}_{s} \subset H_{s}\left(S^{1}\right)$ is an $\mathcal{R}$-invariant Hilbert subspace we have $\bar{\Lambda}_{s}=\bigoplus_{j \in \Gamma} H_{s}\left(S^{1}\right)^{j}$, where $\Gamma \subset \mathbb{Z}_{\geq 0}$. Note that

$$
\left[H_{s}\left(S^{1}\right)^{j}, H_{s}\left(S^{1}\right)^{k}\right]=H_{s}\left(S^{1}\right)^{|j-k|}+H_{s}\left(S^{1}\right)^{j+k}
$$

for $j \neq k$ (this is an elementary calculation). Since the subspace $\bar{\Lambda}_{s} \subset$ $H_{s}\left(S^{1}\right)$ contains a dense subset $\Lambda$ that is closed with respect to commutator operation, for any $j, k \in \Gamma$ such that $j \neq k$ we have $j+k \in \Gamma$ and $|j-k| \in \Gamma$. Hence for the indexing set $\Gamma \subset \mathbb{Z}_{\geq 0}$ we have two possibilities: either $\Gamma=$ $\{0, m\}$ or $\Gamma=m \mathbb{Z}_{\geq 0}$, where $m=G$.C.D. $(\Gamma)$.

Since $v$ is not of type (1.3), $\Lambda$ is not contained in $H_{s}\left(S^{1}\right)^{0}+H_{s}\left(S^{1}\right)^{m}$ for any $m \in \mathbb{Z}_{>0}$. Since $m(v)=1$, we have $m=G$.C.D. $(\Gamma)=1$. Therefore, $\Gamma=\mathbb{Z}_{\geq 0}$ and $\bar{\Lambda}_{s}=H_{s}\left(S^{1}\right)$ for every $s$. Thus, the Lie algebra $\Lambda$ is dense in the Sobolev space $H_{s}\left(S^{1}\right)$ for every $s$. This together with Sobolev's Embedding Theorem [5, p. 411] implies that $\Lambda$ is dense in $\chi\left(S^{1}\right)$. This proves Theorem 2.3 .

Theorem 2.5 Let a family $\mathcal{V}$ of $C^{\infty}$ vector fields on a compact manifold generate a Lie algebra that is dense in the Lie algebra of all the $C^{\infty}$ vector fields. Then the group generated by the flows of the fields from $\mathcal{V}$ is dense in the space of all the $C^{\infty}$ diffeomorphisms isotopic to the identity.

The specialists believe that Theorem 2.5 is known, but we have not found it in literature explicitly.

Proof Let $\Lambda$ denote the Lie algebra generated by $\mathcal{V}$. The group generated by flows of the fields from the family $\mathcal{V}$ is obviously dense in the group generated by flows of the fields from $\Lambda$. Hence, it is dense in the group $G$ generated by flows of all the smooth vector fields. It suffices to show that $G$ is dense.

The authors' original proof of density of the group $G$ was to consider an isotopy of a given diffeomorphism $g$ to the identity and to show directly that $g$ can be approximated by products of flows. To do this, we split the isotopy into small pieces of length $\varepsilon$ : this expesses $g$ as a composition of $N=O\left(\frac{1}{\varepsilon}\right)$ diffeomorphisms $h_{j} \varepsilon$-close to the identity. We approximate each $h_{j}$ by a 
flow; the approximation is of order $\varepsilon^{2}$. The products of approximating flows converge to $g$, as $\varepsilon \rightarrow 0$.

Here we present another argument suggested by Yu.A.Neretin. The subgroup $G$ of the group of diffeomorphisms is normal. But the identity component of the group of diffeomorphisms is simple (Thurston's theorem, see [2, p.24, theorem 2.1.1]. Hence, the group $G$ is dense there. This proves Theorem 2.5 .

Let $G^{0} \subset G$ denote the subset defined by (1.6). It is obviously a subgroup.

Proposition 2.6 The closure in Dif $f_{+}^{\infty}\left(S^{1}\right)$ of the group $G^{0}$ contains the flows of both vector fields $\frac{d}{d x}$ and $v^{\prime}=\left[\frac{d}{d x}, v\right]$.

Proof The group $G^{0}$ itself contains the flow of the constant field $\frac{d}{d x}$, by definition. For every $t \in \mathbb{R}$ the composition $\left(g_{\frac{d}{d x}}^{\frac{1}{N}} \circ g_{v}^{\frac{t}{N}} \circ g_{\frac{d}{d x}}^{-\frac{1}{N}} \circ g_{v}^{-\frac{t}{N}}\right)^{N^{2}}$ belongs to $G^{0}$ and converges in $C^{\infty}$ to the time $t$ flow map of the Lie bracket $v^{\prime}=\left[\frac{d}{d x}, v\right]$, as $N \rightarrow \infty$ (the classical argument). This implies the statement of the proposition.

Corollary 2.7 Let a smooth vector field $v$ on $S^{1}$ be not of type (1.3) and have coprime harmonics: $m(v)=1$. Then the sets $G^{0}$ and $G^{1}$ are dense in Dif $f_{+}^{\infty}\left(S^{1}\right)$.

Proof Recall that by $\mathcal{F} \subset \operatorname{Diff} f_{+}^{\infty}\left(S^{1}\right)$ we denote the group generated by the flows of the fields $\frac{d}{d x}$ and $\left[\frac{d}{d x}, v\right]$. The group $\mathcal{F}$ is dense in Diff $f_{+}^{\infty}\left(S^{1}\right)$. Indeed, it contains the group generated by flows of the fields from the Lie algebra $\Lambda$, by definition and the same classical argument, as in the proof of Proposition 2.6. This together with Theorems 2.3 and 2.5 implies its density. The $C^{\infty}$-closure of the group $G^{0}$ contains all of $\mathcal{F}$, by Proposition 2.6. Hence, $G^{0}$ is dense, as is $\mathcal{F}$. Thus $G^{1}=G^{0} \circ g_{v}^{1}$ is also dense. The corollary is proved.

\subsection{Construction of a family with all phase-lock areas}

Everywhere below, whenever the contrary is not specified, we consider that $v$ is an analytic vector field on $S^{1}$ that is not of type (1.3) and has coprime collection of harmonics: $m(v) \neq 1$. 
Lemma 2.8 For every rational number $\frac{p}{q}$ the set $G_{+}^{1}$ contains a diffeomorphism with rotation number $\frac{p}{q}$ and at least one hyperbolic periodic orbit (either attractor, or repeller).

Proof Fix a hyperbolic diffeomorphism $h: S^{1} \rightarrow S^{1}$ with the rotation number $\frac{p}{q}$. Fix an $l>2$ and a diffeomorphism $g_{0}=g\left(t_{1}^{0}, \ldots, t_{k}^{0}, \tau_{1}^{0}, \ldots, \tau_{k}^{0}\right) \in$ $G^{1}$ that is $C^{l}$-close to $h$ so that $g_{0}$ is also a hyperbolic diffeomorphism with the same rotation number $\frac{p}{q}$. The latter $g_{0}$ exists by Corollary 2.7. Fix a $q$ periodic point $x_{0} \in S^{1}$ of the diffeomorphism $g_{0}$. By definition, $g_{0}^{q}\left(x_{0}\right)=x_{0}$, $\left(g_{0}^{q}\right)^{\prime}\left(x_{0}\right) \neq 1$ (hyperbolicity). Let

$$
T=T\left(t_{2}, \ldots, t_{k}, \tau_{1}, \ldots, \tau_{k}\right)
$$

be the continuous function defined by the equations

$$
g\left(T, t_{2}, \ldots, t_{k}, \tau_{1}, \ldots, \tau_{k}\right)^{q}\left(x_{0}\right)=x_{0}, T\left(t_{2}^{0}, \ldots, t_{k}^{0}, \tau_{1}^{0}, \ldots, \tau_{k}^{0}\right)=t_{1}^{0} .
$$

The function $T$ is analytic, and the corresponding diffeomorphisms $g\left(T, t_{2}, \ldots, t_{k}, \tau_{1}, \ldots, \tau_{k}\right)$ have one and the same rotation number $\frac{p}{q}$. This follows by definition, Proposition 1.10 and implicit function theorem. Recall that $\sum_{j} \tau_{j}^{0}=2 \pi$. The multiplier $\left(g^{q}\right)^{\prime}\left(x_{0}\right)$ is an analytic function of the parameters. It is not identically equal to 1 on the hyperplane $\sum_{j} \tau_{j}=2 \pi$, since it is different from one for $g=g_{0}$ (since $g_{0}$ is hyperbolic). Therefore, for a generic collection of parameter values $t_{2}, \ldots, t_{k}, \tau_{1}, \ldots, \tau_{k}$ in the latter hyperplane with $\tau_{j}>0$ the above multiplier is different from one. By construction, the corresponding diffeomorphism $g$ from (2.3) belongs to $G_{+}^{1}$, has rotation number $\frac{p}{q}$ and the orbit of the point $x_{0}$ is $q$-periodic and hyperbolic. The lemma is proved.

Corollary 2.9 For every rational number $\frac{p}{q}$ there exists an $N=N(p, q) \in$ $\mathbb{N}$ such that for a generic trigonometric polynomial $f(t)$ of degree at most $N$ the family of equations (1.1) has a phase-lock area corresponding to the value $\frac{p}{q}$ of the rotation number.

Proof Given a rational number $\frac{p}{q}$, let $g \in G_{+}^{1}$ be the corresponding diffeomorphism from Lemma 2.8, which has rotation number $\frac{p}{q}$ and a hyperbolic $q$-periodic orbit. For every $k \in \mathbb{N}$ there exists a trigonometric polynomial $f(t)$ such that the time $2 \pi$ flow mapping of the corresponding equation (1.1) with $A=0, B=1$ is arbitrarily $C^{k}$-close to $g$ and the equation has the same rotation number $\frac{p}{q}$ (Proposition 2.11). In particular, one can achieve that the flow mapping has a periodic orbit with non-unit multiplier, as does $g$. 
This means that the parameters $(0,1)$ belong to the interior of the level set $\rho=\frac{p}{q}$ of the rotation number, and hence, family (1.1) has a phase-lock area corresponding to the value $\frac{p}{q}$ of the rotation number. Let $N$ be the degree of the trigonometric polynomial $f(t)$. The latter statement implies that for an open and dense set of trigonometric polynomials $f(t)$ of degree $N$ the corresponding family of equations (1.1) has $\frac{p}{q}$-th phase-lock area. Indeed, let $x_{0}$ be a hyperbolic $q$-periodic point of the time $2 \pi$ flow mapping corresponding to the above parameters $\left(A_{0}, B_{0}\right)=(0,1)$. Consider the continuous function $A=A(B, \phi)$ defined on the product of the set of real numbers $B$ and trigonometric polynomials $\phi$ of degree $N$ by the two following conditions: 1) $x_{0}$ is a $q$-periodic point of the time $2 \pi$ flow map of equation (1.1) with $f$ replaced by $\phi ; 2) A(1, f)=0$. The function $A(B, \phi)$ is well-defined and analytic, by monotonicity and implicit function theorem. The rotation number of the corresponding equations (1.1) with $A=A(B, \phi)$ is constant and equal to $\frac{p}{q}$, by construction and continuity. The multiplier $\mu=\mu(B, \phi)$ at $x_{0}$ of the time $2 \pi q$ flow mapping of the corresponding equation is also an analytic function of $(B, \phi) ; \mu \neq \equiv 1$, since $\mu(1, f) \neq 1$ by construction. Therefore for an open and dense set of trigonometric polynomials $\phi$ of degree $N$ the function $\mu(B, \phi)$ with fixed $\phi$ and variable $B$ is not identically equal to one. This means exactly that the family of equations (1.1) corresponding to $f=\phi$ has $\frac{p}{q}$-th phase-lock area. This proves Corollary 2.9.

Proof of Theorem 1.3. First let us reduce the general case to the case, when $v$ has coprime collection of harmonics. Let $v$ be an arbitrary analytic vector field on $S^{1}$ that is not of type (1.3). In the case, when the collection of its harmonics is not coprime, i.e., $m(v)=m \geq 2$, the field $v$ is invariant under the action of the group $\mathbb{Z}_{m}$ of rotations of order $m$. The quotient projection $S^{1} \rightarrow S_{m}^{1}=S^{1} / \mathbb{Z}_{m} \simeq S^{1}$ transforms $v$ to the quotient vector field $v_{m}$, which is analytic, not of type (1.3) and has a coprime collection of harmonics: passing to the quotient divides the Fourier harmonics by $m$. In what follows we show that for an appropriate analytic function $f: S^{1} \rightarrow \mathbb{R}$ the family of differential equations

$$
\dot{x}=v_{m}(x)+A+B f(t)
$$

on $S_{m}^{1} \times S^{1}$ has phase-lock areas for all the rational values of the rotation number. A phase-lock area corresponding to a rotation number $\rho$ in the family of equations (2.4) is a phase-lock area corresponding to the rotation number $\frac{\rho}{m}$ of the lifted family to $S^{1} \times S^{1}$. This implies that the lifted family also has phase-lock areas for all the rational values of the rotation number. 
Thus, from now on without loss of generality we consider that the field $v$ has a coprime collection of harmonics.

Let us numerate all the rational numbers by natural numbers: $\frac{p_{1}}{q_{1}}, \frac{p_{2}}{q_{2}}, \ldots$ There exists a trigonometric polynomial $f_{1}(t)$ of some degree $N_{1}$ for which the corresponding family of equations (1.1) has a phase-lock area corresponding to $\rho=\frac{p_{1}}{q_{1}}$ (Corollary 2.9). We can approximate it by a trigonometric polynomial $f_{2}$ of certain degree $N_{2}>N_{1}$ such that the corresponding family (1.1) has $\frac{p_{2}}{q_{2}}$-th phase-lock area etc. On each step we choose approximating trigonometric polynomial $f_{j+1}$ so close to $f_{j}$ that the previously constructed phase-lock areas persist and the trigonometric polynomials $f_{j}$ thus constructed converge to an analytic function $f$. The family (1.1) corresponding to the function $f$ has phase-lock areas for all the rational values of the rotation number, by construction. Theorem 1.3 is proved.

\section{Acknowledgements}

We are grateful to V.M.Buchstaber for the statement of problem. We are grateful to him and to E.Ghys, Yu.A.Neretin, J.Rebelo for helpful discussions. We are grateful to the referee and the editors for helpful remarks.

\section{References}

[1] Arnold, V.I. Geometrical Methods in the Theory of Ordinary Differential Equations. Second edition, Springer-Verlag, New York-Berlin-Heidelberg, 1988.

[2] Banyaga, A. The structure of classical diffeomorphism groups, Mathematics and its Applications, 400. Kluwer Academic Publishers Group, Dordrecht; Boston, 1997.

[3] Barone, A.; Paterno, G. Physics and Applications of the Josephson Effect, John Wiley and Sons, New York-Chichester-Brisbane-TorontoSingapore, 1982.

[4] Buchstaber, V.M.; Karpov, O.V.; Tertychnyi, S.I. Effect of quantization of rotation number, Teor. Mat. Fiz., 162:2 (2010), 254-265 (in Russian).

[5] Choquet-Bruhat, Y.; de Witt-Morette, C.; Dillard-Bleick, M. Analysis, Manifolds and Physics, North-Holland, 1977. 
[6] Demazure, M. Classification des algèbres de Lie filtrées, Séminaire N. Bourbaki, 1966-1968, exp. No. 326, 203-213.

[7] Foote, R.L., Geometry of the Prytz Planimeter, Reports on Math. Phys. 42:1/2 (1998), 249-271.

[8] Foote, R.L.; Levi, M.; Tabachnikov, S. Tractrices, bicycle tire tracks, hatchet planimeters, and a 100-year-old conjecture, Amer. Math. Monthly, 103 (2013), 199-216.

[9] Guckenheimer, J.; Ilyashenko, Yu.S. The duck and the devil: canards on the staircase, Moscow Math. J., 1 (2001), No. 1, 27-47.

[10] Ilyashenko, Yu.S. Lectures of the summer school "Dynamical systems", Poprad, Slovak Republic, 2009.

[11] Ilyashenko, Yu.S.; Filimonov, D.A.; Ryzhov, D.A. Phase-lock effect for equations modeling resistively shunted Josephson junctions and for their perturbations, Functs. Analiz i iego pril., 45 (2011), No. 3, 41-54 (in Russian). English translation in Funct. Analysis and its appl. 45 (2011), no. $3,192-203$.

[12] Josephson, B.D., Possible new effects in superconductive tunnelling, Phys. Lett., 1 (1962), No. 7, 251-253.

[13] Lie, S. Theorie des Transformationsgruppen, Math. Ann. 16 (1880), 441-528; see also: Sophus Lie's 1880 transformation group paper, Math Sci Press, Brookline, Mass., 1975.

[14] McCumber, D.E. Effect of ac impedance on dc voltage-current characteristics of superconductor weak-link junctions, J. Appl. Phys., 39 (1968), No.7, 3113-3118.

[15] Rebelo, J.C.; Silva, R.R. The multiple ergodicity of non discrete subgroups of Dif $f^{\omega}\left(S^{1}\right)$, Moscow Math. J., 3 (2003), No. 1, 123-171.

[16] Schmidt, V.V., Introduction to physics of superconductors (in Russian), MCCME, Moscow, 2000.

[17] Stewart, W.C., Current-voltage characteristics of Josephson junctions. Appl. Phys. Lett., 12 (1968), No. 8, 277-280. 